\title{
Cognitive and Action-Based Aspects of Developing Decisions in Parietal Cortex
}

\author{
(D)Jan Kubanek ${ }^{1,2}$ and $\mathbb{C}^{-E r i c ~ M o o s h a g i a n ~}{ }^{1}$ \\ ${ }^{1}$ Department of Anatomy and Neurobiology, Washington University School of Medicine, St. Louis, Missouri 63110, and ${ }^{2}$ Department of Biomedical \\ Engineering, Washington University in St. Louis, St. Louis, Missouri 63130 \\ Review of de Lafuente et al.
}

Neurons in many regions of the brain encode variables that provide information about the choice a decision-maker is about to make. For instance, during perceptual decisions, neurons in the lateral intraparietal area (LIP), the frontal eye fields, the superior colliculus, and the dorsolateral prefrontal cortex of the monkey encode the amount of sensory evidence accumulated in support of a saccadic eye movement to one of two targets shown on a screen (Gold and Shadlen, 2007). Decision scientists have long speculated about the nature of the decision-related activity in these brain regions. These circuits may constitute a general cognitive, amodal decision network that computes a decision before it is passed on to motor circuitry for execution (Gottlieb and Balan, 2010). Alternatively, decisions may evolve in specific circuits depending on the effector used to communicate the decision (Cui and Andersen, 2007). The specialization of a particular circuit for a particular effector may increase the speed and accuracy of decisions such as where to look, where to head, or which item to reach for.

In their recent article published in The Journal of Neuroscience, de Lafuente et al. (2015) contribute to this debate by

Received March 26, 2015; revised April 19, 2015; accepted April 22, 2015.

Correspondence should be addressed to Dr. Jan Kubanek, Department of Anatomy and Neurobiology, Washington University School of Medicine, 660 South Euclid Avenue, St Louis, MO 63110. E-mail: jan@eye-hand.wustl.edu.

DOI:10.1523/JNEUROSCI.1181-15.2015

Copyright $\odot 2015$ the authors $\quad 0270-6474 / 15 / 358382-02 \$ 15.00 / 0$ investigating the neuronal correlates of developing decisions in distinct effector contexts. Monkeys chose one of two visual targets based on the quality of sensory evidence in a perceptual stimulus. Crucially, animals communicated their decision using one of two instructed actions-an eye movement (saccade) or an arm movement (reach). The use of two distinct response actions allowed the authors to distinguish whether a developing decision in a particular brain region was specific to a particular action, or whether it was generic, independent of which action was to be performed. The authors recorded discharge activity of neurons on the lateral bank (LIP) and the medial bank (MIP) of the intraparietal sulcus in posterior parietal cortex while the monkeys performed the task. Based on previous studies in which animals were instructed to make a saccade or a reach to a visual target, one would expect decisions communicated using a saccade to modulate LIP activity more strongly than decisions communicated using a reach (Snyder et al., 1997; Dickinson et al., 2003; Cui and Andersen, 2007). In contrast, based on previous studies, one would expect neurons in the posterior part of MIP [the parietal reach region (PRR)] to show the opposite tendency (Snyder et al., 1997; Calton et al., 2002; Cui and Andersen, 2007).

de Lafuente et al. (2015) found that the discharge rate of neurons in both LIP and MIP was correlated with the amount of evidence accumulated over time to sup- port a particular choice (de Lafuente et al., 2015, their Fig. 8). The effects closely reproduced those previously observed in LIP using saccade tasks (Gold and Shadlen, 2007). In particular, activity was modulated by the animals' choice, i.e., whether an animal was going to choose a target placed in the neuronal response field or the opposite target. Furthermore and importantly, activity was modulated by the amount of decision evidence in the stimulus in support of choosing a particular target. Crucially, the decision-related modulation in MIP was modulated by the animals' choice and by the sensory evidence much more strongly during choices made using reaches than for choices made with saccades. This finding suggests that decisions in MIP specifically evolve in the context of an upcoming reach movement.

Unexpectedly, however, stronger choicerelated modulations during reach choices were also observed in LIP (de Lafuente et al., 2015, their Fig. 8c). A similar observation is made when the saccade and reach responses were compared in an instructed delay task that the authors used to decide which neurons to record in the main task (Fig. 1). The generally enhanced representation of reach movements raises the question of whether the two kinds of responses in this study were comparable at the behavioral level. For instance, plans to make a reach might have formed earlier than plans to make a saccade (de Lafuente et al., 2015, their Fig. 6b), which could 
LIP neuronal responses in an instructed delay task of de Lafuente et al., 2015

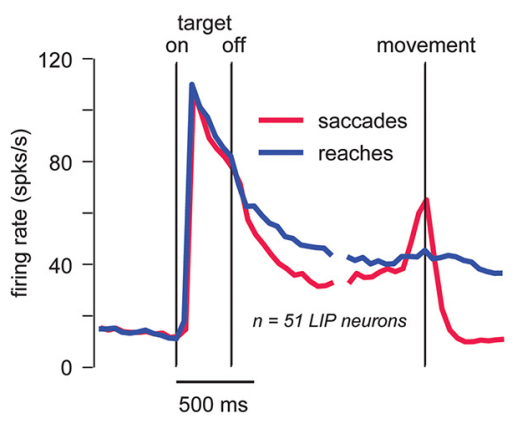

Figure 1. Mean neuronal responses of $51 \mathrm{LIP}$ neurons recorded by de Lafuente et al. (2015) in an instructed movement task. The figure shows all trials in which the animals were required to make a saccade (red) or a reach (blue) into the neuronal response field. In other studies (Snyder et al., 1997; Dickinson et al., 2003; (ui and Andersen, 2007), LIP neurons exhibit, during the delay period, greater activity in saccade trials compared with reach trials. The present study shows a marked opposite trend. The figure contrasts Figure 4, c and d, of de Lafuente et al. (2015), with permission.

have resulted in an activation advantage for reaches.

Using the same instructed delay task, previous studies found that plans to make a saccade activate LIP neurons at least as strongly as plans to make a reach (Snyder et al., 1997; Dickinson et al., 2003; Cui and Andersen, 2007). In a marked contrast, the present study shows a much higher activity in LIP for reach trials compared with saccade trials, a difference that at a midpoint through the trial amounts to an average of almost 20 spikes/s ( $n=51$ LIP cells). In addition to instructed movement tasks, it has been shown that LIP neurons also exhibit stronger choicerelated modulations during saccade compared with reach choices (Cui and Andersen, 2007; Kubanek and Snyder, 2015). Yet, the present study shows the opposite (de Lafuente et al., 2015, their Fig. 8, bottom). These marked discrepancies could be due to several factors. First, training animals to reach to a target without looking at the target is difficult, and it is possible that following this training, animals still form a partial plan to make a saccade to the target. A simultaneous recruitment of both saccade and reachsensitive neurons in LIP may then amplify LIP activity during such partially dissociated reaches. Second, it is possible that the present study used different criteria for selecting which neurons to record from compared with previous studies. This is supported by the observation (Fig. 1) that the average response to the target onset in this study is $>100$ spikes/s, which is much higher than found in previous studies (Snyder et al., 1997; Dickinson et al., 2003; Cui and Andersen, 2007). Third, it is possible that a portion of the cells included in the present study in the LIP pool were in fact recorded from MIP. A mislabeling of this sort can occur because the lateral (LIP) and medial (MIP) banks of the IPS are in close proximity (e.g., de Lafuente et al., 2015, their Fig. 3c, bottom). Therefore, a clear anatomical delineation is difficult to ascertain. To overcome this difficulty, researchers typically supplement the anatomical information with responses of parietal neurons in the above-mentioned instructed delay task before collecting data in a main task. This approach was taken here. Nonetheless, the authors performed this mapping task only on a subset $(99 / 148)$ of the neurons. This inconsistent functional mapping amplifies the question of a proper attribution of neurons into a particular area. Because MIP neurons are strongly reach-selective, including just a proportion of MIP cells in the LIP pool could fundamentally affect the LIP result.

Regardless of the LIP discrepancies, de Lafuente et al.'s (2015) study contributes to the field in two important ways. First, the study identifies a putative evidenceaccumulation process in two parietal areas during decisions communicated using arm movements. This finding extends the previous findings made in LIP in eye movement contexts (Gold and Shadlen,
2007) to arm movement contexts. The finding that evidence accumulation is generally stronger in both areas during reach movements needs to be carefully investigated in the future, and in doing so, some of the above points need to be considered. Second, the study finds that MIP is strongly reach-specific during evidence accumulation. Along with other findings (Cui and Andersen, 2007; Kubanek and Snyder, 2015), this suggests that MIP (and its posterior part, PRR) specifically represents decisions concerned with where to reach. This finding supports the notion of an intentional representation of decisions in parietal cortex. In this framework, decisions run on circuits devoted to planning and execution of a specific action. This neural architecture may be useful to mediate fast and accurate decisions such as which item to reach for when faced with alternatives.

\section{References}

Calton JL, Dickinson AR, Snyder LH (2002) Non-spatial, motor-specific activation in posterior parietal cortex. Nat Neurosci 5:580588. CrossRef Medline

Cui H, Andersen RA (2007) Posterior parietal cortex encodes autonomously selected motor plans. Neuron 56:552-559. CrossRef Medline

de Lafuente V, Jazayeri M, Shadlen MN (2015) Representation of accumulating evidence for a decision in two parietal areas. J Neurosci 35: 4306-4318. CrossRef Medline

Dickinson AR, Calton JL, Snyder LH (2003) Nonspatial saccade-specific activation in area LIP of monkey parietal cortex. J Neurophysiol 90:2460-2464. CrossRef Medline

Gold JI, Shadlen MN (2007) The neural basis of decision making. Annu Rev Neurosci 30:535574. CrossRef Medline

Gottlieb J, Balan P (2010) Attention as a decision in information space. Trends Cogn Sci 14:240-248. CrossRef Medline

Kubanek J, Snyder LH (2015) Reward-based decision signals in parietal cortex are partially embodied. J Neurosci 35:4869-4881. CrossRef Medline

Snyder LH, Batista AP, Andersen RA (1997) Coding of intention in the posterior parietal cortex. Nature 386:167-170. CrossRef Medline 